\title{
2012 Colorado alphaherpesvirus latency symposium
}

\author{
Randall J. Cohrs • Don Gilden
}

Received: 17 August 2012 /Accepted: 24 August 2012 /Published online: 13 September 2012

(C) Journal of NeuroVirology, Inc. 2012

\section{Colorado alphaherpesvirus latency symposium}

The second symposium of the Colorado Alphaherpesvirus Latency Society (CALS) convened May 16-18, 2012, at the Christiania Lodge in Vail, Colorado. The CALS mission is to provide a venue whereby researchers actively studying alphaherpesvirus latency can discuss current findings and future experimental approaches in a relaxed setting. In attendance were 46 clinicians/scientists who collectively traveled 83,000 miles to provide 29 presentations focused on various aspects of type 1 herpes simplex virus type (HSV-1), varicella zoster virus (VZV), and simian varicella virus (SVV) latency. The participants (Fig. 1) who assembled in the picturesque town of Vail in the heart of the Colorado Rocky Mountains accounted for more than 191 PubMed-cited publications in the past year. Discussions at the second CALS have already led to collaborations that promise to advance the field. A brief summary of each presentation follows.

\section{Reports}

Bernard Roizman, University of Chicago, presented initial studies using trigeminal ganglionic (TG) organ cultures to study HSV reactivation. TG were excised 30 days after corneal inoculation of mice and immediately incubated in a medium containing anti-nerve growth factor (NGF) antibody to initiate reactivation or containing NGF and

\footnotetext{
R. J. Cohrs • D. Gilden $(\bowtie)$

Department of Neurology, School of Medicine,

University of Colorado Denver,

Mail Stop B182, 12700 E. 19th Avenue,

Aurora, CO 80045, USA

e-mail: don.gilden@ucdenver.edu

D. Gilden

Department of Microbiology, Denver School of Medicine, University of Colorado,

Aurora, CO, USA
}

epidermal growth factor (EGF) to suppress reactivation. Viral RNA was first detected 5 to $9 \mathrm{~h}$ after excision, followed by appearance of viral DNA. Reactivation occurred within the time span of the HSV-1 replicative cycle and did not require treatment of ganglia with antiviral drugs that induce single-chain termination. There were two key findings: (1) Representative members of the four kinetic classes of viral genes $\left(\alpha, \beta, \gamma_{1}\right.$, and $\left.\gamma_{2}\right)$ were expressed simultaneously and transcribed in the presence of cycloheximide, indicating that VP16 is not required to initiate viral gene expression, and (2) pro-apoptotic drugs accelerated reactivation of the virus from TG incubated in a medium containing NGF plus EGF. Apoptosis induced by damage to neurons may trigger reactivation. The organ culture model holds promise as a means to study HSV reactivation from latency.

Xiaoli Yu, University of Colorado, has developed an in vitro model of VZV infection of human neurons. Highly pure $(>95 \%)$ terminally differentiated human neurons derived from induced pluripotent stem cells (from Cellular Dynamics International) were infected with cell-free VZV (Zostavax vaccine). At 14 days post-infection, cells appeared normal compared to human fetal lung fibroblasts (HFL) which developed a cytopathic effect (CPE) less than a week after VZV infection. Although the tissue culture medium from VZV-infected neurons did not produce a CPE in uninfected HFL, cocultivation of infected neurons with uninfected HFLs produced a CPE in uninfected HFLs. Quantitative PCR using VZV primers for ORFs 4, 11, 28, 62, and 68 confirmed the presence of the VZV genome. Transcripts corresponding to multiple VZV immediate-early, early, and late genes were detected in the nonproductively infected neurons. Immunofluorescence staining also revealed VZVspecific proteins in nonproductively infected neurons. VZV IE 62 was found in the nucleus, while VZV IE 63 was seen in both the nucleus and cytoplasm, but predominantly in the cytoplasm of the infected neurons. VZV-infected neurons provide a unique in vitro system to study the VZV-neuronal relationship and the potential to investigate mechanisms of VZV reactivation. 

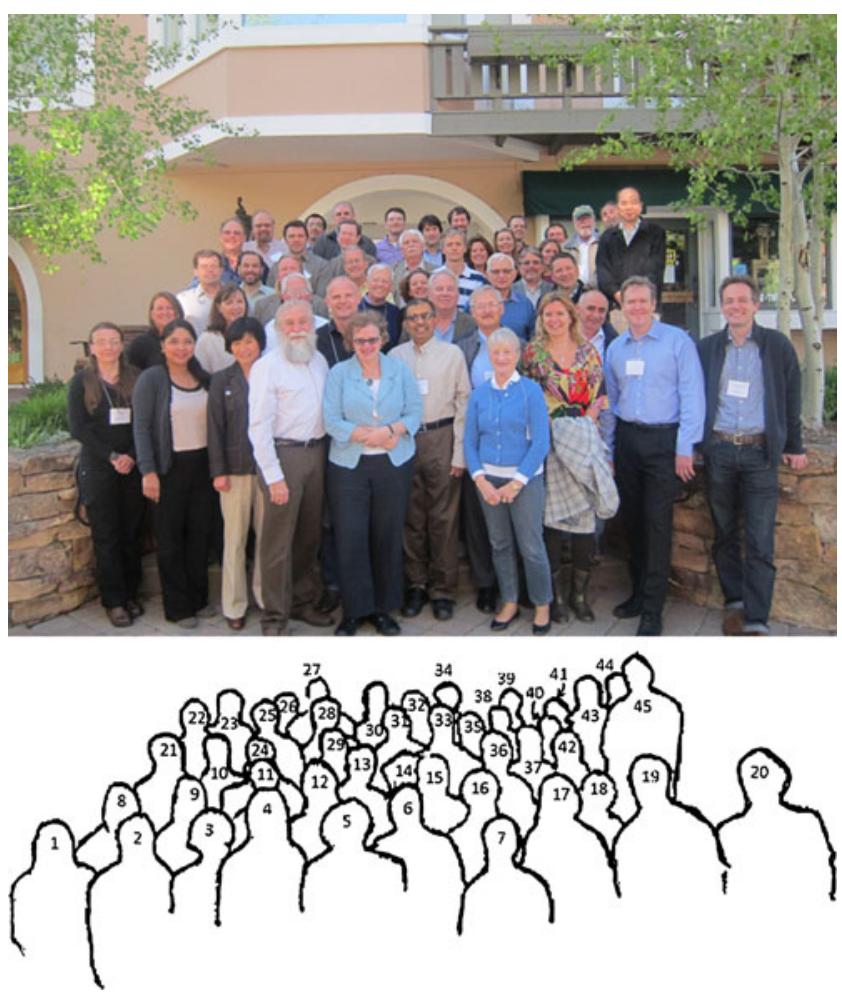

Fig. 1 Left to right 1 Martine Aubert, 2 Maria Nagel, 3 Xiaoli Yu, 4 Randy Cohrs, 5 Judy Breuer, 6 Ravi Mahalingam, 7 Anne Gershon, 8 Leigh Zerboni, 9 Stephanie James, 10 Ian Mohr, 11 Robert Hendricks, 12 Klaus Osterrieder, 13 Don Gilden, 14 Ilhem Messaoudi, 15 Bryan Cullen, 16 Nigel Fraser, 17 Marieke Verweij, 18 Francis Carbone, 19 Dorian McGavern, 20 Tobias Derfuss, 21 Dale Carpenter, 22 Kip Kinchington, 23 Scott Schmid, 24 David Bloom, 25 Igor Jurak, 26 Marius Birlea, 27 Clinton Jones, 28 Keith Jerome, 29 Daniel Carr, 30 Matthew Taylor, 31 Charles Grose, 32 John Blaho, 33 Luis Schang, 34 Rafael Harpaz, 35 Andrea Bertke, 36 Bernard Roizman, 37 Joel Rovnak, 38 Nancy Sawtell, 39 David Davido, 40 Nicole Giordani, 41 Stacey Efstathiou, 42 Patrick Lomonte, 43 Richard Thompson, 44 Angus Wilson, 45 Jason Chen, 46 Michael Gershon (not shown)

Stacey Efstathiou, Cambridge University, has utilized HSV-1 recombinants expressing Cre recombinase under the control of both lytic and latent promoters to activate a loxP-flanked LacZ gene in ROSA26R reporter mice. A fluorescent reporter gene facilitated the isolation of single neurons, which in combination with quantitative real-time PCR, enabled an estimation of the latent viral genome copy number. Analysis revealed high cellular fluorescence when using the strong CMV immediate-early promoter, and all fluorescent cells contained HSV-1 DNA. The latent HSV-1 genome copy number varied from 1 to $\sim 1,000$ copies per cell (mean, 160), with no bystander fluorescence observed. HSV-1 copy number in neurons in which Cre recombinase was driven by the ICP0 and thymidine kinase promoters did not differ from that in neurons without Cre recombinase, consistent with the view that heterogeneity of latent virus DNA copy number reflects axonal trafficking of virions from the periphery rather than limited virus gene expression before establishment of latency. Future work using latently infected cells that underwent prior lytic cycle promoter activation will focus on determining whether they represent cells with enhanced reactivation competence and whether lytic cycle gene products can impact the biology of latently infected neurons.

Patrick Lomonte, Université Lyon, presented a study of HSV-1 latency analyzed at the single neuron level using fluorescent in situ hybridization (FISH). During acute infection, after which HSV-1 becomes latent in TG neurons, the switch between the lytic cycle and latency is characterized by a change in viral gene transcription. Nuclear architecture, including nuclear domains, is a determinant for control of gene expression and is likely to influence the fate of viral infection. FISH analysis, developed to examine single TG neurons in mice 4 to 14 days post-infection (dpi), revealed viral genomes in neurons and satellite cells at 4-6 dpi but only in neurons after 6 dpi. In satellite cells, viral genomes exhibited a replicative-like pattern, whereas neurons showed both a replicative-like and an acute infection multi-spot pattern until 6 dpi. From 6-14 dpi, the replicative-like pattern gradually disappeared, while the multi-spot pattern evolved to single or double spots. Expression of ICP4 and ICP27 and nuclear translocation of cellular NFkB were detected only in cells with the replicative-like pattern. The multi-spot pattern co-localized with the PML-NB proteins PML, Daxx, and ATRX along with SUMO-1 and SUMO-2/3. Whereas PML, Daxx, and ATRX were still visibly associated with viral genomes at $14 \mathrm{dpi}$, SUMO-1 and SUMO-2/3 disappeared at 8 dpi. Analysis of TG proteins at 6 dpi showed that PML, Daxx, and ATRX significantly increased according to their interferon-responsive gene status. Overall, these data show that HSV-1 genome patterns evolve from acute infection toward latency and that PML-NB proteins are involved in acquisition and maintenance of latent HSV-1 patterns.

Luis Schang, University of Alberta, described dynamics of histone mobilization during HSV-1 infection, with implications for latency and reactivation. After discussing previous models proposing that HSV-1 DNA is assembled in chromatin during latency but not during lytic infections, he presented data on the unstable nature of the nucleosome-like nucleoprotein complexes formed with HSV-1 DNA during lytic infection. The complexes were unstable before and after onset of HSV-1 DNA replication, but stability was greater before HSV-1 DNA replication or when replication was inhibited. These results are consistent with previous data showing that HSV-1 DNA was more efficiently protected from nuclease digestion during latency, when there is no HSV-1 DNA replication. For chromatin to assemble with HSV-1 genomes in latently infected cells, histones would have to be mobilized from cellular chromatin to interact with the HSV-1 DNA before replication. Core and linker histones were mobilized before replication, as determined 
by fluorescence after photobleaching. Mobilization of a core histone variant $(\mathrm{H} 3.1)$, shown by others to interact with HSV-1 DNA only after HSV-1 DNA replication, was enhanced when HSV-1 DNA replication was inhibited, suggesting that it is normally mobilized before the replication and becomes immobilized by its assembly into HSV-1 chromatin during HSV-1 DNA replication. Mobilization of another variant (H3.3), reported to interact with the viral DNA before it begins to replicate, was not affected by inhibition of HSV-1 DNA replication, suggesting that it is mobilized from cellular chromatin before HSV-1 DNA replication begins and then immobilized by DNA replicationindependent assembly in HSV-1 chromatin. This latter variant is enriched in neurons, most of which show no significant replication of HSV-1 genomes during the establishment of latency. Dr. Schang proposed that HSV-1 genomes are in nucleosome-like nucleoprotein complexes in both lytic and latent infections, but that the nucleoprotein complexes formed with HSV-1 DNA are much more stable during latent infection. It remains to be determined whether differential stabilities of the HSV-1 DNA nucleoprotein complexes are a consequence of or, instead, underlie the differences between lytic and latent infections in transcription and DNA replication.

David C. Bloom, University of Florida, investigated regulation of HSV latent gene expression in neurons of spinal nerve ganglia by the cellular insulator protein CTCF. Latent gene expression is tightly repressed except for the latencyassociated transcript (LAT). Chromatin immunoprecipitation (ChIP) analysis has demonstrated that the LATencoding region is associated with histones containing transcriptionally permissive $\mathrm{N}$-terminal modifications, whereas lytic gene regions are associated with transcriptionally repressive ones. This suggests that the genome is organized into separate chromatin domains with different degrees of transcriptional permissiveness. The HSV-1 genome contains six unique clusters of binding sites for CTCF, which participates in formation of chromatin boundaries on cellular chromosomes. Using ChIP, he demonstrated that the predicted CTCF motifs are bound by CTCF during latency. Finally, high-resolution ChIP-sequence analysis of the latent HSV genome indicated that CTCF acts as a classical boundary that separates regions containing histones with different transcription potential.

Nancy Sawtell, Cincinnati Children's Hospital Medical Center, described studies addressing the regulation of HSV latency and reactivation in vivo. A central feature of HSV biology is long-term maintenance of the viral genome in ganglionic neurons. Periodic activation of the viral lytic cycle underlies spread of infection to new hosts, recurrent disease, and chronic inflammation in the nervous system. The measured frequency of reactivation in mice is 1 per 120,000 latently infected neurons per day, with similar estimates in humans. Stressful stimuli such as fever increase reactivation frequency in humans, and hyperthermic stress increases the rate of reactivation to $\sim 1 / 2,500$ latently infected neurons. Thus, reactivation is maintained at a frequent steady rate at the host level but is very rare at the cellular level. The mechanisms governing these events are central to HSV biology and pathogenesis. HSV utilizes the tegument protein VP16 to transactivate (in a complex with two cellular proteins) its IE genes to initiate the viral lytic cycle. Whereas reactivation has long been considered to occur via a VP16-independent mechanism, Dr. Sawtell has discovered a distinct regulatory strategy through which VP16 (normally a late gene) is expressed very early to initiate the lytic cycle in neurons. The VP16 gene promoter regions identified are consistent with multi-component positive/negative feedback elements and signature neuronal stress response regulatory modules. A second dimension to this model is provided by the demonstration that VP16 function is regulated through phosphorylation, which modulates coactivator partnering and thus transcriptional activation by VP16. Phosphorylation of VP16 and a potentially competing posttranslational modification are strategically integrated into stress-responsive neuronal physiology. Thus, the regulation of VP16 at both the transcriptional and functional levels may form a stratified regulatory circuit that is intimately wired into the host stress response to effectively balance latency and reactivation.

Dale Carpenter, University of California, Irvine, extended his report at last year's CALS that HSV-1 LAT has a positive effect on virus reactivation from latently infected mouse trigeminal ganglia. To identify regions of HSV-1 LAT responsible for enhanced reactivation, two mutated viruses were constructed. The first HSV-1 mutation used the LAT promoter to express only the first 1,499 bp of LAT. The second HSV-1 mutation deleted the first 1,578 bp of LAT and resulted in transcription of the LAT $3^{\prime}$ end by the LAT promoter. Only the second virus mutation significantly enhanced virus reactivation. Previous studies hypothesized the existence of a protein with properties similar to those of HSV-1 ICP0 encoded in the large open reading frame (ORF) located in the 2.0-kb LAT intron. Since this LAT-associated ORF is contained in the second mutated virus, the data support the hypothesis that expression of a LAT-encoded ICP0 homolog may increase the efficiency of HSV-1 reactivation.

Richard Thompson, University of Cincinnati, investigated the requirement of HSV VP16 promoter elements to initiate reactivation of latently infected neurons in vivo. Most humans worldwide have been infected by HSV, after which the virus becomes latent in ganglionic neurons. No effective vaccine is available, and no therapy eliminates latency or prevents reactivation. With the long-term goal of finding interventions for recurrent HSV episodes by 
defining mechanisms that control establishment of and reactivation from latency, Dr. Thompson's studies have focused on the role of the virion transactivating protein VP16 in the balance between lytic and latent infection of neurons in vivo. Results to date support the hypothesis that VP16 expression and transactivation activity are critical to the balance between the establishment of latent infection and viral replication in trigeminal ganglion neurons. Use of combined histochemical and immunohistochemical approaches with defined viral mutants to investigate regulation of VP16 expression in neurons in vivo showed that: (1) the VP16 promoter upstream of the TATA box contains multiple redundant elements that direct appropriate late viral gene kinetics on VP16 expression; (2) the region near the TATA box and the 5'UTR of the VP16 gene contains specialized elements that regulate de novo expression of VP16 in sensory neurons; (3) this regulatory region plays a dominant role in the balance between latent and lytic infection of neurons, but is not required for proper VP16 expression in other cells or tissues; and (4) after latent infection is established, this same unique VP16 promoter region is required at the earliest stage of viral reactivation from latency.

Ian Mohr, New York University, investigated the control of HSV-1 latency in neurons by axonal mTOR signaling and the 4E-BP translation repressor. PI3-kinase/Akt signaling stimulated by binding of NGF to the TrkA receptor is essential to maintain HSV-1 latency in primary neuron cultures. The diverse consequences of NGF on target neurons in culture are known to require new protein synthesis and treatment of latently infected neurons with a pulse of puromycin, a reversible inhibitor of polypeptide elongation that results in premature chain termination and more efficient reactivation. Analyses to examine how ongoing neuronal protein synthesis controls HSV-1 latency focused on mTORC1, a critical signaling mode through which capdependent translation is regulated in response to numerous environmental stimuli, and on 4E-BP1, a repressor of capdependent translation that is hyperphosphorylated and inactivated upon activation of mTORC1. Inhibition of mTORC1 by treatment with rapamycin, exposure to hypoxia, or RNAi-mediated depletion of the raptor regulatory subunit all resulted in HSV-1 reactivation in the presence of NGF. Furthermore, sustained mTORC1 activation by lentiviral transduction of a mutant Rheb allele, which constitutively stimulates mTORC1, inhibited reactivation induced by targeting molecules positioned upstream (PI3 kinase or Akt), but not downstream (mTORC1), of Rheb. Remarkably, expression of a constitutively active 4E-BP1 translational repressor, but not wild-type 4E-BP1, in latently infected neuronal cultures was sufficient to stimulate reactivation. Finally, inhibiting mTOR in axons induced reactivation. Together, these results demonstrate that: (1) mTORC1mediated inactivation of a cellular cap-dependent translation repressor is required to maintain HSV-1 latency in this cultured neuron system and (2) local changes in axonal mTOR signaling which controls translation regulate latent HSV1 genomes in a spatially segregated compartment. By relying on mTORC1-stimulated cap-dependent translation, latent HSV-1 is able to respond to a diverse array of physiological cues that potentially impact the host neuron.

Randall Cohrs, University of Colorado, presented a model to investigate alphaherpesvirus reactivation. Both HSV-1 and VZV become latent in human trigeminal ganglia (TG), but only HSV-1 reactivates in explanted TG. Recovery of infectious virus in explanted TG depends on virus DNA replication. Using replication of virus DNA as a marker of virus reactivation, Dr. Cohrs tested human TG that were dissociated and distributed in multiple wells of 96-well tissue culture plates. Real-time PCR analysis of HSV-1 and VZV DNA in each well at 0,5 , and 10 days postexplant showed that HSV-1 and, to a lesser extent, VZV DNA replication occurred. Future experiments will identify treatments, e.g., hypoxia, dexamethasone, heat, and superinfection that might induce virus DNA replication.

Angus Wilson, New York University, presented a detailed temporal analysis of HSV-1 reactivation in cultured neurons. Latency is dependent on continuous NGF signaling mediated through PI3 kinase, Akt, and mTORC1. Use of a selective inhibitor of PI3 kinase triggered synchronous reactivation and revealed two distinct waves of viral mRNA accumulation. The first wave (phase I) involved promiscuous transcription of viral lytic genes independent of viral protein synthesis or viral DNA replication, consistent with transient de-repression of the latent episome. The second wave (phase II) of HSV-1 gene transcription resembled the classic productive replication cascade and coincided with viral genome amplification and infectious virion synthesis. Viral proteins expressed in phase I included VP16, which unexpectedly remained in the neuronal cytoplasm until phase II when it accumulated in the nucleus of some, but not all, responsive neurons. This mirrors PI3 kinasedependent relocalization of the cellular VP16-accessory factor HCF-1. Use of a mutant virus (in1814) or shRNA against VP16 stimulated phase II, but not phase I, supporting the notion that VP16-mediated transactivation is a critical determinant of the HSV-1 latency/reactivation switch and that the location of VP16 within neurons is tightly regulated, presumably by host factors. It is conceivable that virus genomes remaining in phase I re-establish latency and that lytic proteins expressed in phase I provide the antigens detected by $\mathrm{CD} 8+\mathrm{T}$ cells associated with latently infected neurons in ganglia.

Clinton Jones, University of Nebraska, presented results of studies on bovine herpesvirus $1(\mathrm{BHV}-1)$ protein expression in neurons during reactivation from latency. BHV-1 can be induced to reactivate from latency by a single injection of 
the synthetic corticosteroid dexamethasone. Within $90 \mathrm{~min}$, ICP0 and VP16 protein expression were detected in trigeminal ganglionic neurons. Neurons that contained ICP0 frequently expressed VP16. In contrast, other late viral proteins ( $\mathrm{gC}$ or $\mathrm{gD}$ ) were rarely detected and not until $6 \mathrm{~h}$ after treatment. These studies suggest that many neurons express viral regulatory proteins (VP16 and ICP0) during early phases of reactivation, whereas the virus reactivates from only a few neurons to produce an infectious virus.

Andrea Bertke, University of California, San Francisco, described HSV-1 infection of specific types of neurons in primary adult mouse TG neuronal cultures. The TG contain a heterogeneous population of neurons. Neurons identified by monoclonal antibody A5 support HSV-1 latent infection in vivo, but only $2 \%$ of A5+ neurons become productively infected by HSV-1 in dissociated adult TG neurons in vitro. However, up to $45 \%$ of $\mathrm{KH} 10+$ neurons, a different population of neurons, become productively infected with HSV1, although $\mathrm{KH} 10+$ neurons do not support latent infection in vivo. Since neuron selectivity is regulated at or before immediate-early (IE) gene expression, HSV-1 IE gene deletion viruses were analyzed in $\mathrm{A} 5+$ and $\mathrm{KH} 10+$ neurons. HSV-1 with an ICP4 deletion failed to grow in either A5+ or $\mathrm{KH} 10+$ neurons, while HSV-1 with an ICP0 deletion productively infected $\mathrm{KH} 10+$ but not $\mathrm{A} 5+$ neurons, similar to wild-type HSV-1. However, an HSV-1 virus with an ICP27 deletion (d27-1) grew to higher titers in cultured neurons than parental KOS1.1 HSV-1, and A5+ and KH10 + neurons were both permissive for productive infection with $\mathrm{d} 27-1$. Thus, ICP27 restricts productive infection in ganglionic neurons, specifically in A5+ neurons, the principal site of HSV-1 latency. Furthermore, more virus antigenpositive neurons were seen in murine dorsal root ganglia after sciatic nerve injection with $\mathrm{d} 27-1$ than after infection with parental KOS 1.1 HSV-1. Use of various ICP27 deletion mutants localized the regions of ICP27 that appeared to limit productive infection in $\mathrm{A} 5+$ neurons to the $\mathrm{C}$-terminal domain and to the N-terminal nuclear export and localization signals. Additionally, an ICP22-deletion virus (d22) yielded a phenotype in cultured neurons like that of $\mathrm{d} 27-1$. Overall, ICP27 is essential for productive infection in dividing cells but inhibits productive infection of $\mathrm{A} 5+$ neurons. ICP22, although not essential for productive infection in dividing cells, also restricted infection in A5+ neurons. These findings suggest that HSV-1 IE proteins ICP27 and ICP22 play an important role in the establishment of latency.

Leigh Zerboni, Stanford University, examined 27 ganglia from 18 individuals using rabbit anti-IE63 IgG antibody and matched preimmune IgG. Previous reports indicated that IE63 expression is rare. Recently, the Arvin lab reported that animal-derived antibody preparations may produce false-positive cytoplasmic staining of VZV proteins in neurons from individuals with blood type $\mathrm{A} 1$, due to the presence of contaminating endogenous rabbit or mouse antihuman blood type A1 antibodies that react within Golgi zones, findings confirmed by Ouwendijk and colleagues. The Arvin laboratory further extended these observations by demonstrating that contaminating antihuman blood group A1 antibodies were also present in a mouse antiIE63 monoclonal antibody (clone 9A12). Overall, these findings suggest that differences in antibody preparations might underlie the wide discrepancies in the reported frequency of detection of VZV protein expression in neurons during latency. Immunoreactivity to putative VZV latencyassociated proteins may be explained by blood type Arelated staining artifacts.

Matthew Taylor, Princeton University, described models to quantify alphaherpesvirus virions associated with anterograde sensory axon-to-cell spread. HSV and pseudorabies virus (PRV) strains expressing one of three fluorophores (mCeruelean, EYFP or mRFP) fused to a nuclear localization sequence (XFP-NLS) were constructed and characterized. A compartmentalized neuronal culture system that reconstitutes anterograde spread was infected with three equivalent strains of either HSV or PRV, and cellular fluorescence was imaged after axonal spread. While the levels of anterograde axonal spread differed between HSV and PRV, on average, less than two genomes were expressed in each newly infected cell after axonal spread. To further characterize this limitation, axonal egress at a single virion level was visualized using a capsid-tagged virus (VP26-mRFP fusion); nearly $50 \%$ of cells infected by axonal spread contained only a single capsid. These complementary analyses indicate a restriction in the number of virions involved in neuron-to-cell spread at the point of axonal egress. Such restriction may limit the overall diversity of the progeny herpesvirus population during propagation of viral infections within and between hosts.

Charles Grose, University of Iowa, has studied autophagy in VZV-infected cells in tissue culture. While previous electron microscopy analyses revealed sites of virus assembly and envelopment, re-examination also revealed doublewalled vesicles characteristic of autophagosomes. These vesicles contained amorphous debris and did not surround intact viral particles. Laser scanning confocal microscopic analysis revealed autophagosomes in skin samples obtained from humans with varicella and zoster and immunolabeled with monoclonal antibodies to individual VZV proteins as well as a rabbit polyclonal antibody directed against the LC3 protein found in the autophagosome wall. In infected cells in tissue culture, VZV IE62 is located in the nucleus early in infection and in the cytoplasm later; in biopsied skin cells, autophagosome formation was found in cells containing IE62 localized to the nucleus, indicating that autophagy is an early event in VZV infection. Overall, autophagy is common during both varicella and zoster. Importantly, the 
VZV genome lacks the HSV counterpart of the autophagyinhibiting protein ICP34.5.

Ilhem Messaoudi, Oregon National Primate Research Center, investigated the role of simian varicella virus (SVV) ORF 61 protein during infection of rhesus macaques (RMs). SVV infection of RMs recapitulates hallmarks of infection with $\mathrm{VZV}$, an exclusively human neurotropic alphaherpesvirus that causes chicken pox during primary infection and becomes latent in ganglia. Thus, studies of SVV infection promise to shed light on the poorly understood host and viral factors that control establishment and maintenance of VZV latency. Analyses focused on the role of SVV ORF 61, a viral transactivator that encodes the most frequently detected transcript during SVV latency in RMs, in the balance between lytic and latent SVV infection. Comparison of disease progression, viral replication, immune response, and the establishment of latency after infection with a recombinant SVV lacking ORF 61 (SVV $\Delta$ ORF61) versus wild-type SVV revealed no significant differences in varicella development, viral loads, viral replication kinetics, or in the ability to establish latency in ganglia, when viral loads were similar. Interestingly, the adaptive immune response in RMs infected with SVV $\Delta$ ORF61 was dampened, with decreased $\mathrm{B}$ cell and $\mathrm{T}$ cell proliferative bursts, lower SVV-specific IgM and IgG antibody titers, and a lower frequency of SVV-specific T cells. Mechanisms underlying the diminished adaptive immune response were investigated by measuring changes in plasma cytokine levels as well as frequencies of dendritic cells (DCs), which play a key role in priming the immune response. Analysis revealed alterations in recruitment and proliferation of DC populations as well as reduced inflammatory cytokine levels in the plasma. Since ORF61 is a transactivator, potential differences in viral transcript levels in SVV $\Delta$ ORF61-infected animals were also investigated; SVV transcripts were less abundant during the acute phase of infection in SVV $\Delta$ ORF61-infected RMs. Together, these data suggest that the absence of ORF61 leads to reduced viral transcriptional activity, which in turn results in lower antigen load and decreased DC activation and recruitment, culminating in a lower adaptive immune response.

Marieke Verweij, Oregon Health and Science University Vaccine and Gene Therapy Institute, showed that the JAKSTAT signaling pathway was inhibited by the SVV-encoded ORF63. SVV infection of rhesus macaques has been characterized as the first robust animal model for VZV, recapitulating key hallmarks of human infection. Dr. Verweij's use of this model to study virus-host interactions has identified and explored SVV-associated immune modulatory functions in vitro with the ultimate goal of testing their relevance for SVV pathogenesis. Studies using a luciferase reporter system demonstrated that SVV infection of rhesus fibroblasts (RF) abrogates type I interferon (IFN)-dependent gene transcription. Correspondingly, IFN-dependent proteins that confer antiviral activity such as MX-1, ISG15, and ISG54 were not expressed during SVV infection following exposure to IFN. Analysis of the IFN-dependent JAK-STAT signaling pathway showed that SVV prevents phosphorylation of STAT2 and subsequent nuclear localization of the ISGF3 transcriptional complex (consisting of STAT1, STAT2, and IRF9). Since previous work showed that a mutant VZV lacking ORF63 was hypersensitive to IFN exposure, a tet-inducible adenovirus was constructed to express SVV ORF63 protein in RFs. Expression of SVV ORF63 inhibited IFN-dependent antiviral gene expression at levels comparable to those observed in SVV-infected cells, although expression of SVV ORF63 alone did not prevent IFN-induced STAT2 phosphorylation. These data indicate that SVV encodes multiple ORFs that inhibit IFN-induced JAK-STAT signaling, including ORF63 and at least one yet unidentified protein.

Judy Breuer, University of London, provided estimates of VZV evolution in humans based on phylogenetic analysis of conserved viral genes from alphaherpesviruses that infect multiple animal species. Published data suggest a mutation rate of $\sim 10^{-7}$, with a divergence of viral clades 50,000 to 100,000 years ago. These data are consistent with the outof-Africa theory of VZV evolution and geographical segregation of clades currently apparent. However, molecular clock methods based on Baysian analysis date the separation of clades to less than 500 years ago. To reconcile these diverse estimates, Dr. Breuer sequenced vaccine virus as well as virus from patients who developed varicella and zoster immediately after vaccination. Based on vaccination and sampling dates for each virus, mutation rates for zoster were calculated to be $\sim 100$ times less than for varicella. She hypothesized that dating the divergence of VZV clades is influenced by the time between virus infection and the onset of varicella or zoster, a function of population densities.

Keith Jerome, Fred Hutchinson Cancer Research Center and University of Washington, introduced the concept of using rare-cutting endonucleases to target latent virus DNA for mutagenesis and inactivation. After describing the three main classes of rare-cutting endonucleases (zinc finger nucleases, homing endonucleases, and TAL effecter nucleases), he presented data demonstrating that homing endonucleases (HEs) can effectively target and induce mutation of episomal targets without damaging the host cell. DNA sequencing revealed various mutations induced in episomal targets, predominantly small deletions of 1 to 23 nucleotides but sufficient to eliminate production of protein encoded by target sequences. Similar results were also obtained using viral targets that were integrated in the human genome. He provided an overview of the methodology by which HE specificity is redirected toward new sequences and concluded with several examples of redesigned HEs specific for novel viral targets.

Martine Aubert, Fred Hutchinson Cancer Research Center, presented studies on the application of rare-cutting 
endonucleases (HE) to cure chronic/latent viral infection. The approach consists of targeting latent HSV DNA for mutagenesis following introduction of DNA double strand break by HE. HEs are highly specific because they recognize long DNA sequences (on average, 20 nucleotides). In collaboration with Cellectis (Paris, France), Dr. Aubert used HSV1-specific HE (HSV-HE) that had been modified to recognize a unique DNA sequence present in HSV-1 but not in the human genome. Primary human fibroblasts containing quiescent HSV-1 DNA were treated with HSV-HE in the presence of the $3^{\prime}->5^{\prime}$ DNA exonuclease Trex 2 showed reduced HSV-1 reactivation. Amplicon sequencing from treated cells demonstrated mutations in the HSV-1 sequence targeted by HSV-HE. These studies support further work to develop a cure for HSV-infected individuals by targeted mutagenesis of virus DNA in latently infected neurons.

Robert Hendricks, University of Pittsburgh, presented data demonstrating that immunodominant $\mathrm{CD}^{+} \mathrm{T}$ cells specific for the HSV $\mathrm{gB}_{498-505}$ epitope are fully functional in TG latently infected with HSV-1. In contrast, TG-resident $\mathrm{CD} 8^{+} \mathrm{T}$ cells that are specific for subdominant HSV epitopes exhibit a loss of functionality during latency. Treatment of mice with antibody to the IL-10 receptor (IL-10R) selectively increased subdominant $\mathrm{CD} 8^{+} \mathrm{T}$ cells in latently infected $\mathrm{TG}$, as well as their expression of interferon-gamma and granzyme $\mathrm{B}$, and reduced virus reactivation in ex vivo TG cultures. These findings suggest that expanding the repertoire of functional $\mathrm{HSV}$-specific $\mathrm{CD}^{+} \mathrm{T}$ cells in latently infected TG reduces HSV reactivation from latently infected ganglia.

David Davido, University of Kansas, investigated disruption of nuclear domain 10 (ND10) structures by HSV-1 ICP0. HSV-1 ICP0 is an immediate-early phosphoprotein with E3 ubiquitin ( $\mathrm{Ub}$ ) ligase activity that transactivates viral gene transcription, in part by disrupting ND10. Previous studies suggest that phosphorylation regulates ICP0 functions. Mutation of putative phosphorylation sites within a region (termed Phos 1) that lies in the N-terminal portion of ICP0 impairs E3 Ub ligase and ND10-disrupting activities of ICP0 and diminishes viral replication in cell culture and in vivo. To identify specific phosphorylation site(s) responsible for the Phos 1 phenotypes, putative phosphorylation sites were mutated to alanine (S224A, T226A, S224A/T226A, T231A, and T232A). These ICP0 mutants were examined for their ability to dissociate ND10 constituents and support HSV-1 replication. Results showed that the S224A, S224A/T226A, T231A, and T232A mutations were unable to efficiently or completely dissociate the cellular protein PML from ND10s, while the S224A/T226A mutation was defective in its ability to dissociate the cellular protein Sp100 from ND10s. S224A and S224A/T226A mutations appear to impair transactivation by ICP0. When the E3 Ub ligase activity of ICP0 was examined, S224A and
S224A/T226A mutants were more stable than wild-type ICP0, suggesting their limited ability to auto-ubiquitinate. One ICP0 ubiquitination target (USP7) was more stable in the presence of these two ICP0 mutants. Introduction of S224A and S224A/T226A mutations into the HSV-1 genome diminished virus replication. Overall, specific phosphorylation sites within the Phos 1 region differentially regulate ICP0 activities, which is required for efficient viral replication.

Paul Kinchington, University of Pittsburgh, reported on an animal model of allodynia (hypersensitivity to tactile stimuli) induced by VZV. Allodynia is a common feature of postherpetic neuralgia (PHN) and is difficult to treat. Rats inoculated with VZV in the footpad developed allodynia and heat-sensitivity for 8-12 weeks, as measured by paw withdrawal to a light stimulus. In rats showing behavioral pain responses, footpad inoculation of replication-defective HSV expressing the opioid modulator of pain under the control of the CMV immediate-early promoter reduced both mechanical allodynia and thermal hyperalgesia induced by VZV within 1 day. Pain relief lasted up to 4 weeks. The results suggest the potential use of HSV vector delivery of a proenkephalin to treat zoster and PHN.

Anne Gershon, Columbia University, reviewed recent evidence obtained by her and colleagues Jason Chen and Michael Gershon indicating that VZV becomes latent in the enteric nervous system (ENS) in most individuals with a history of varicella or vaccination. She presented clinical data supporting the notion that the presence of VZV DNA in saliva, previously reported in astronauts in association with space flight and in individuals with acute varicella or zoster, indicates active VZV infection and thus can be used as a diagnostic aid. She and colleagues confirmed that VZV DNA could not be detected in saliva of patients after zoster. VZV DNA was detected in the saliva of a patient with severe unilateral allodynia and suspected zoster sine herpete; after treatment with valacyclovir, the patient's pain resolved, and VZV DNA disappeared from the saliva. The final patient described, a 16-year-old male with massive gastric bleeding necessitating partial resection of an ulcerated stomach, showed salivary VZV DNA of the vaccine type (vOka) after surgery; the patient never had chicken pox, but had received varicella vaccine twice. The surgical specimen also contained VZV DNA and detectable VZV gE and ORF63 proteins in the mucosa, especially at the ulcer margins. A mucosal infiltrate of CD4 and CD8 cells was prominent close to the ulcer. No surviving neurons were found in the region of the gastric myenteric plexus. The patient recovered, and mucosal morphology was normal in a repeat biopsy 3 weeks later, which revealed neither VZV DNA nor VZV proteins. This case is the first documentation of enteric zoster, although post-vaccination vOka latency in the ENS has been reported. Published case reports have also linked 
VZV to achalasia, gastric ulcers, and intestinal pseudoobstruction; however, prior episodes of enteric VZV infection occurred in a setting of concomitant skin rash. Diagnosis of VZV in the absence of rash is difficult. Salivary confirmation of VZV infection would be useful in diagnosing zoster sine herpete and enteric zoster, both of which may be far more common than currently thought. Further studies are needed to verify the diagnostic value of testing salivary DNA for VZV infection.

Tobias Derfuss, University of Basel, reported results of a study examining the immune response against VZV and subclinical viral reactivation during treatment of multiple sclerosis (MS) patients with fingolimod. Reactivation of latent VZV is known to be influenced by immunosuppression. Fingolimod is a novel selective immunosuppressant that acts on the sphingosine receptor pathway and renders lymphocytes insensitive to a signal necessary for egress from lymph nodes. In treated patients, naïve and central memory lymphocytes are decreased in blood. A longitudinal follow-up of MS patients before and during fingolimod therapy showed that VZV-specific, IFN $\gamma$-producing cells were also reduced during treatment. The absolute number of lymphocytes activated and proliferating upon VZV stimulation was diminished in the blood of fingolimod-treated patients. Quantitative PCR analysis of VZV- and EBV-DNA copy numbers in saliva and blood revealed no positive blood samples, whereas $20 \%$ of fingolimod-treated patients showed viral copies in saliva compared to $2 \%$ of patients without immunosuppression and healthy controls. These data suggest that fingolimod might compromise antiviral immunity.

Daniel Carr, University of Oklahoma, described a new area in HSV-1 pathology, i.e., virus-induced lymphangiogenesis. He reported that HSV-1 infection induces lymphatic vessel development before blood vessel development in the avascular cornea. The mechanism of induction was unique in that leukocytes were not involved in the initial process, although the event required local production of VEGF-A acting via the VEGF receptor-2, but not the VEGF receptor-3, pathway. Dr. Carr presented recent findings in floxed VEGF-A mice infected with an ICP0-Cre-expressing HSV-1 recombinant, which ablated VEFG-A expression in the cornea after ocular infection in floxed mice, whereas parental SC16 HSV-1 did not. These findings further substantiate that most VEGF-A expressed early in the cornea after HSV-1 infection is produced by infected (epithelial) cells. The loss of corneal VEGFA expression in floxed VEGF-A mice infected with the $\mathrm{Cre}$ expressing virus was associated with a significant reduction in neovascularization in the cornea at the time of viral latency (day 30 post-infection).

Acknowledgments The Colorado Alphaherpesvirus Latency Society thanks the following companies for the generous support: Merck Vaccines, Diagenode, Inc., Fisher Scientific, Mary Ann Liebert, Inc., Miltenyi Biotec, and the VZV Foundation. We especially thank the owners of the Christiania Lodge at Vail. 